\title{
Colostomy closure: how to avoid complications
}

\author{
Andrea Bischoff • Marc A. Levitt • Taiwo A. Lawal • \\ Alberto Peña
}

Published online: 17 August 2010

(C) The Author(s) 2010. This article is published with open access at Springerlink.com

\begin{abstract}
Purpose Colostomy is an operation frequently performed in pediatric surgery. Despite its benefits, it can produce significant morbidity. In a previous publication we presented our experience with the errors and complications that occurred during cases of colostomy creation. We now have focused in the morbidity related to the colostomy closure. The technical details that might have contributed to the minimal morbidity we experienced are described. Methods The medical records of 649 patients who underwent colostomy closure over a 28 -year period were retrospectively reviewed looking for complications following these procedures. Our perioperative protocol for colostomy closure consisted in: clear fluids by mouth and repeated proximal stoma irrigations $24 \mathrm{~h}$ prior to the operation. Administration of IV antibiotics during anesthesia induction and continued for $48 \mathrm{~h}$. Meticulous surgical technique that included: packing of the proximal stoma, plastic drape to immobilize the surgical field, careful hemostasis, emphasis in avoiding contamination, cleaning the edge of the stomas to allow a good 2-layer, end-to-end anastomosis with separated long-term absorbable sutures, generous irrigation of the peritoneal cavity and subsequent layers with saline solution, closure by layers to avoid dead space, and avoidance of hematomas. No drains and no nasogastric tubes were used. Oral fluids were started the day after surgery and patients were discharged $48-72 \mathrm{~h}$ after the operation.
\end{abstract}

\footnotetext{
A. Bischoff $(\square) \cdot$ M. A. Levitt · T. A. Lawal · A. Peña Division of Pediatric Surgery, Colorectal Center for Children, Cincinnati Children's Hospital Medical Center, 3333 Burnet Avenue, ML 2023, Cincinnati, OH 45229, USA

e-mail: andrea.bischoff@cchmc.org
}

Results The original diagnoses of the patients were: anorectal malformation (583), Hirschsprung's disease (53), and others (13). 10 patients (1.5\%) had complications: 6 had intestinal obstruction (5 due to small bowel adhesions, 1 had temporary delay of the function of the anastomosis due to a severe size discrepancy between proximal and distal stoma with a distal microcolon) and 4 incisional hernias. There were no anastomotic dehiscences or wound infection. There was no bleeding, no anastomotic stricture and no mortality.

Conclusion Based on this experience we believe that colostomy closure can be performed with minimal morbidity provided a meticulous technique is observed.

Keywords Colostomy · Anorectal malformation · Colonic surgery

\section{Introduction}

Colostomy is an operation frequently performed in pediatric surgery, especially for cases of anorectal malformations and Hirschsprung's disease. Despite its benefits, it can produce significant morbidity and mortality.

According to the literature, anastomotic dehiscence consecutive to colostomy closure in the pediatric population can occur with a frequency that varies from 0 to $12.5 \%$; and wound infection from 0.4 to $45 \%$ [1-16]. Other complications such as bleeding $[3,14]$, anastomotic stricture $[2,13,15]$, and death $[7,9,12]$ have also been reported in the pediatric population.

In a previous publication, we presented our evaluation of the errors and complications that occurred during colostomy creation in a large series of referred patients [17]. In this current review we chose to share our preoperative, 
intraoperative, and postoperative routines for colostomy closure, with special emphasis on the surgical technique, which we believe plays an important role in achieving low morbidity rates.

\section{Methods}

The medical records of 649 patients who underwent colostomy closure over a 28-year period (1982-2010) were retrospectively reviewed looking for complications following these procedures. All these cases were performed by the senior author.

Our perioperative protocol for colostomy closure consisted in: (1) admission on the day before surgery; (2) clear liquids by mouth; (3) repeated proximal stoma irrigations with saline solution, $24 \mathrm{~h}$ prior to the operation; (4) administration of IV antibiotics during anesthesia induction and continued for $48 \mathrm{~h}$; (5) meticulous surgical technique that included: packing of the proximal stoma, plastic drape to immobilize the surgical field, multiple silk sutures in the mucocutaneous junction of the stomas to provide uniform traction that allows the surgeon to identify the correct dissection plane, remaining as close as possible to the bowel wall (Figs. 1, 5), careful hemostasis, emphasis in avoiding contamination, cleaning the edge of the stomas to allow a precise anastomosis (Figs. 2, 5); a two-layer, end-to-end anastomosis with separated long-term 6-0 absorbable sutures (Figs. 3, 5), generous irrigation of the peritoneal cavity and subsequent layers with saline solution, closure in layers to avoid dead space, avoidance of hematomas, and wound coverage with collodium (Figs. 4, 5). In the postoperative, no nasogastric tubes were used, and the patients received clear fluids on the first postoperative day, if they were not distended or nauseated. Most of the patients were discharged home on the second or third day following the operation.

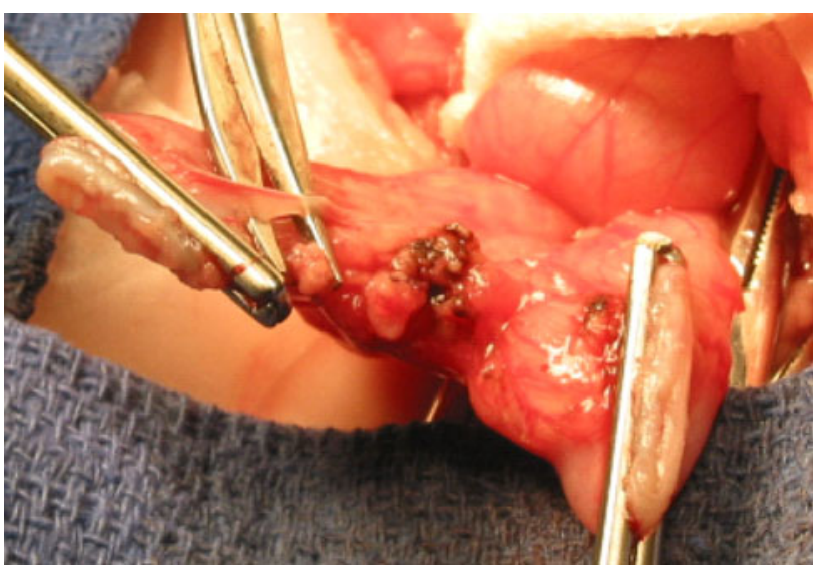

Fig. 2 Cleaning the edges of the stomas, preparing for the anastomosis

\section{Results}

The original diagnoses of the patients were: anorectal malformation (583), Hirschsprung's disease (53), and others (13) that included: malignancy (4), teratoma (3), ulcerative colitis (2), pelvic trauma (2), vaginal atresia (1), and giant seminal vesicle (1).

Of the 649 colostomies that we closed, only 148 were opened by us, the remaining 501 patients had their colostomies opened elsewhere. The type of colostomy was: separated stoma (480), loop (137), and Hartman (32). The encountered size discrepancy during colostomy closure varied from no size discrepancy to $5: 1$.

Ten patients (1.5\%) had complications: 6 had intestinal obstruction (5 due to small bowel adhesions and 1 had temporary delay of the function of the anastomosis due to a severe size discrepancy between proximal and distal stoma with a distal microcolon), and there were 4 incisional hernias. There were no anastomotic dehiscences, wound
Fig. 1 Multiple silk sutures in the mucocutaneous junction that allow for a uniform traction. The dotted line shows the elliptical incision. The opening is performed layer by layer
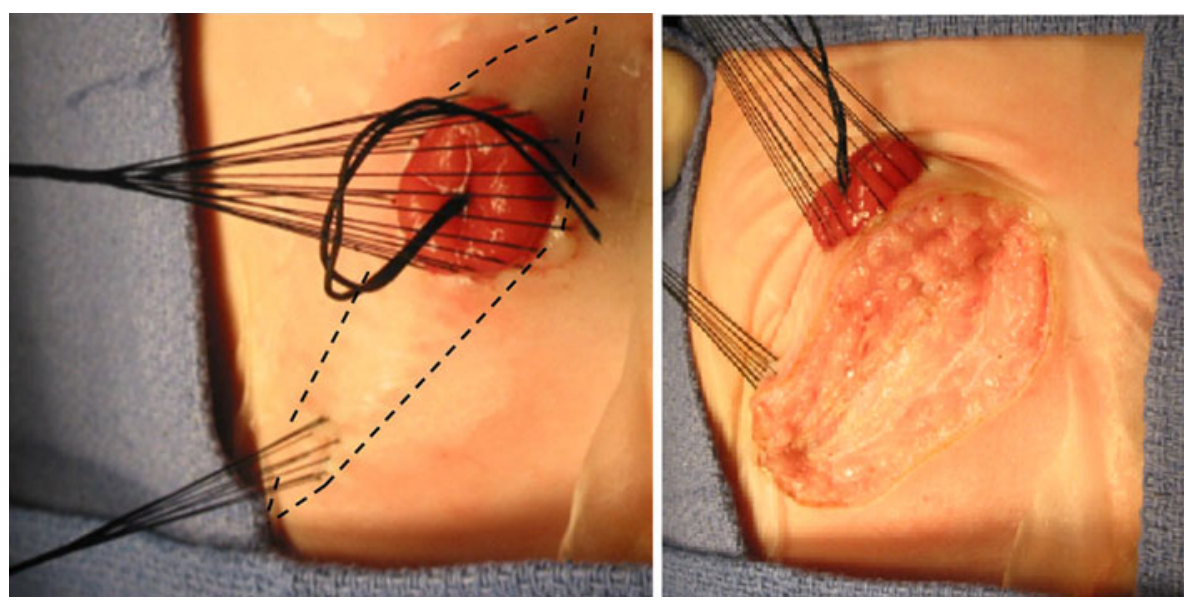
Fig. 3 Two-layer anastomosis: a external layer of posterior wall, $\mathbf{b}$ internal layer of posterior wall, c internal layer of anterior wall, $\mathbf{d}$ external layer of anterior wall
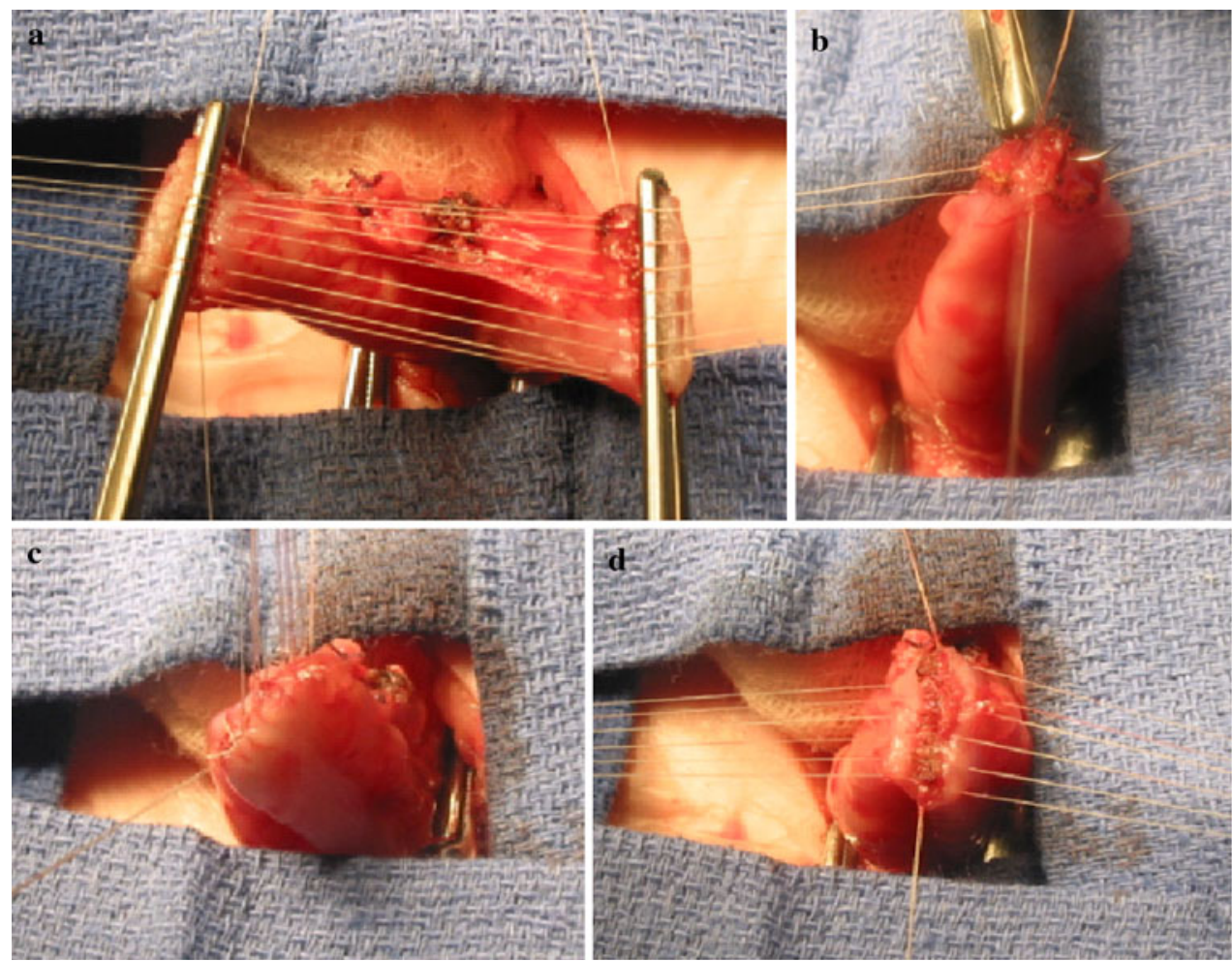

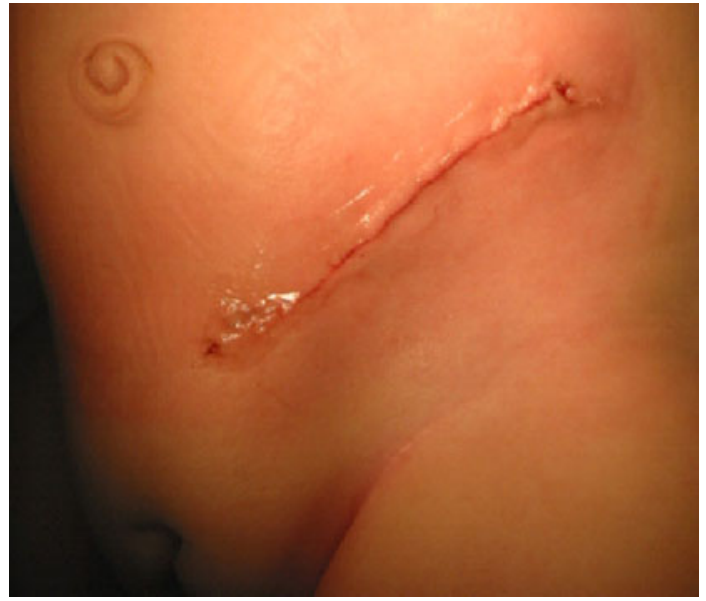

Fig. 4 Closed wound, covered with colodium

infections, episodes of bleeding or anastomotic stricture. There were no mortalities.

\section{Discussion}

Colostomy closure is a routine procedure performed frequently by pediatric surgeons all over the world. It is an elective procedure that is assumed to be easy, reproducible, and with minimal or no morbidity. Yet, the literature indicates that this procedure still may be the source of significant complications, including death. Because of these facts, as well as the very good results encountered in our series, we decided to share our routines and surgical technique that we use during the colostomy closure procedure.

From all the routines and technical steps that we follow, we do not know which ones are fundamental and which ones are not as vital. We just know that by following these routines, we did not have a single case of wound infection, abscess, hematoma, seroma, or dehiscence of the wound or the anastomosis.

Irrigation of the proximal stoma the day before surgery, as well as the administration of only clear fluids by mouth, may or may not contribute to our results; sometimes, during the operation, we find a completely clean proximal stoma whereas, other times we find stool, yet the results have been equally good in both circumstances.

During the first few years, we administered erythromycin by mouth to our patients, as described in the literature as an adjunct for bowel preparations [18]. It provoked frequent vomiting and therefore, we stopped using it.

Something similar happened with the use of prophylactic antibiotics. Early in our series, we administered ampicillin, gentamicin and clindamycin; subsequently, we switched to ampicillin, gentamycin and Flagyl; and lately we use a cephalosporin and Flagyl. All these changes were a result of the recommendation of the infectious disease department of the Hospitals where we worked. Yet, we observed no difference in our infection rate.

On the other hand, we feel that our operative routines are very important to achieve our results. 


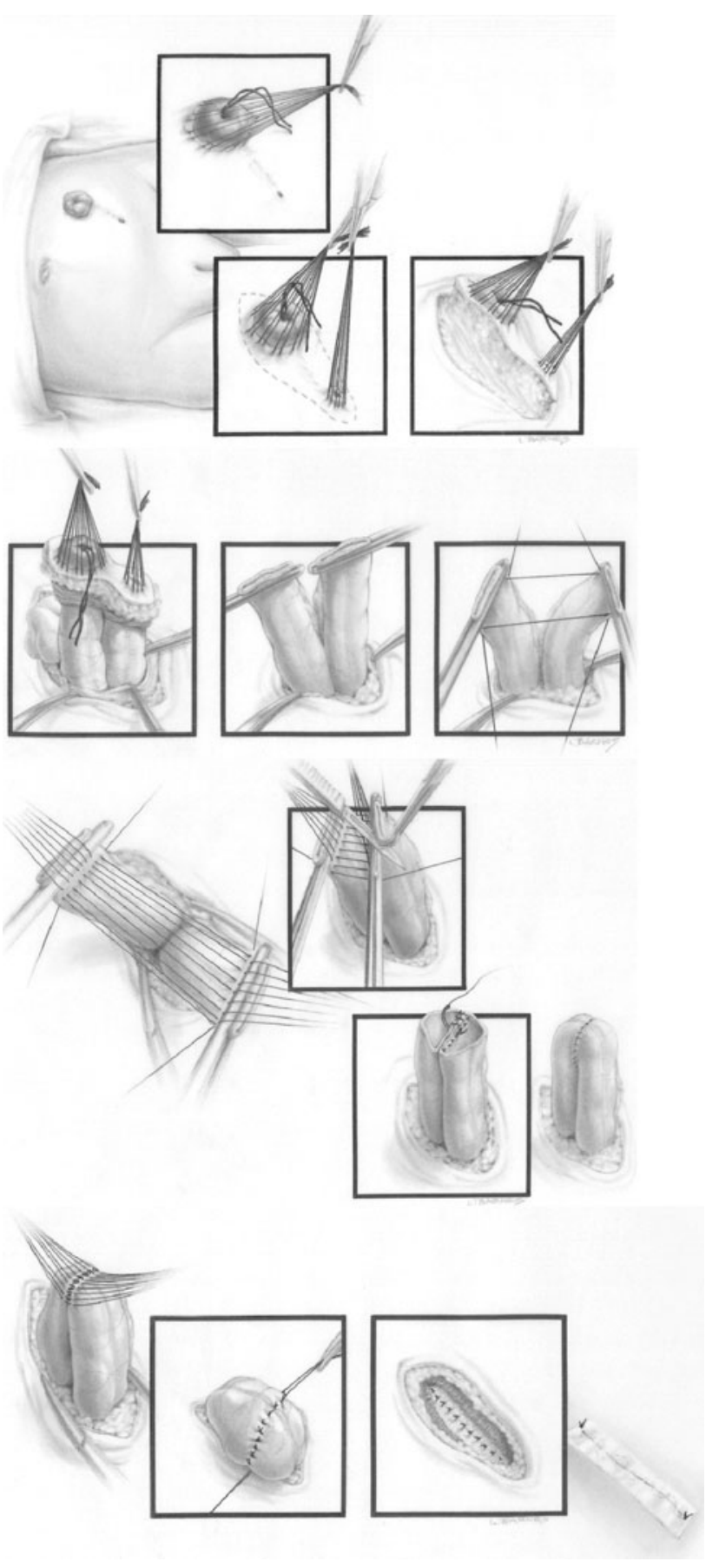

Fig. 5 Artistic diagram of the colostomy closure technique

We prepare the skin with Betadine and alcohol, and pack the proximal stoma with gauze impregnated with Betadine. The operative field is immobilized with a plastic drape. This last maneuver, we believe, is very important. We have been impressed by the high frequency of gross contamination of the operative field that occurs when the towels that surround the operative area are not fixed. We have seen this repeatedly when observing operations done by others or even in the slides presented in meetings. The gross contamination is easily seen; but despite it, some surgeons ignore it.

We strongly recommend using coagulation to coagulate and cutting current to cut, it might seem unnecessary to mention, but many surgeons use either coagulation or blend current all the time. This, we believe, causes excessive burning that may leave damaged tissue, which contributes to bacterial proliferation. We spend significant time performing a meticulous selective hemostasis, which results in blood losses difficult to quantify, since they are minimal.

There are several articles indicating that a one-layer bowel anastomosis is as good as a two-layer one [19-21]. However, we have made interesting observations: during morbidity and mortality meetings when we hear about an anastomotic leakage or dehiscence that occurred in patients that underwent a one-layer bowel anastomosis; often when describing the secondary operation, a two-layer anastomosis was used.

Profuse irrigation of the peritoneal cavity as well as each one of the layers of the wound, we believe, is also important.

A single-layer abdominal wall closure with a running suture is fashionable, quick and easy. However, we feel that it produces more pain; it is cosmetically undesirable and more prone to end up in evisceration. Again in morbidity and mortality sessions, whenever there is a wound dehiscence, it seems that during the secondary operation interrupted stitches are used.

In addition to closing each one of the layers of the abdominal wall separately, we put special emphasis in obliterating all spaces; we close the Scarpa fascia with interrupted stitches and use another layer of sutures for the dermis to decrease the tension between the skin edges, allowing for a safer closure and to achieve a better cosmetic scar.

We are now convinced and agree with others that a nasogastric tube is unnecessary most of the times, when a clean operation has been done with minimal bowel manipulation and a bloodless field [22, 23].

The problem of an incisional hernia was detected months after the operation, and represent a lack of closure of one of the layers of the wound. This is a preventable complication and it reinforced our conviction of the need to pay attention to all the steps and details of the operation.

Most of the times when we close a colostomy, we find a size discrepancy between the proximal and distal bowel. The greater the discrepancy, the more technically demanding the procedure. We try, in most cases, to perform an end-to-end anastomosis, however, when the size discrepancy is greater than $4: 1$, we prefer to do an end-toside anastomosis, which works equally well and is technically easier to perform (Fig. 6).

It is well known that the size discrepancy is more dramatic the longer the waiting time with the colostomy open. 
Fig. 6 End-to-side anastomosis for size discrepancy greater than $4: 1$. Window-type of stoma created about $5-10 \mathrm{~cm}$ proximal to the anastomosis
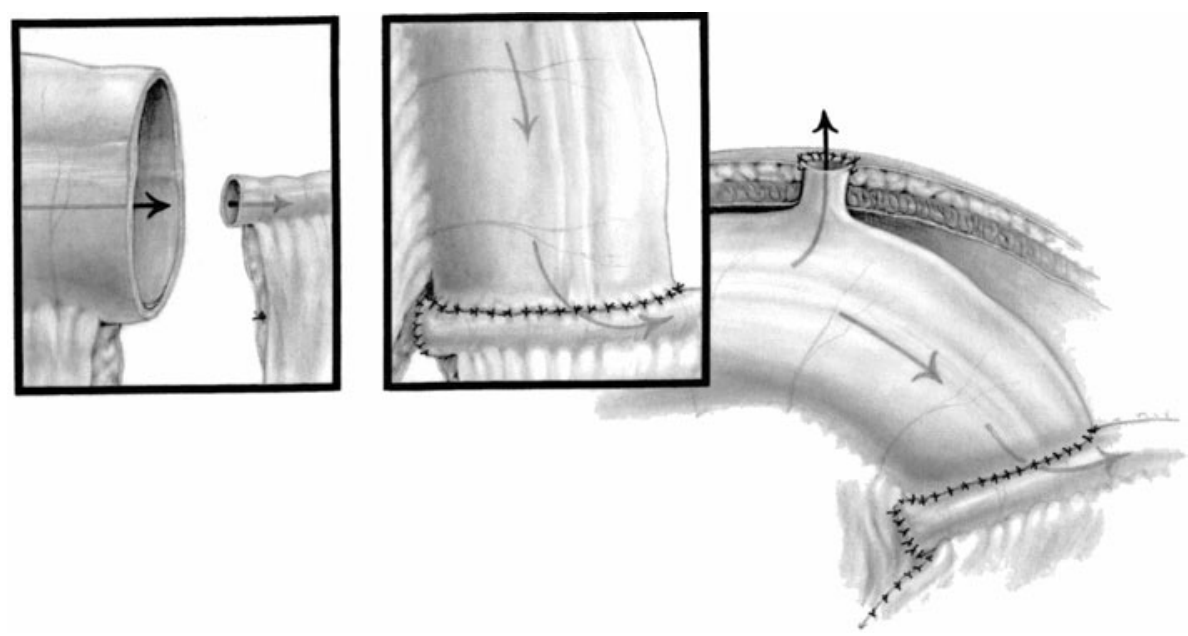

We operated on some patients who had an open colostomy for ten years. The size discrepancy in that type of case can be as much as 10:1, with the distal microcolon. In one of those cases, the anastomosis did work, but it took a longer period of time to open up than it usually does.

More recently, when confronted with that kind of technical challenge, we have been using a technique that we found to be useful in cases of colonic atresia, in which the size discrepancy is dramatic. The technique consists in performing an end-to-side anastomosis plus a window-type of stoma created about $5-10 \mathrm{~cm}$ proximal to the anastomosis (Fig. 6). During the first few postoperative days, one can see a large fecal output through the window; eventually the output decreases and the amount of stool passing through the downstream bowel increases, until the window closes, the anastomosis is efficient, and the microcolon grows.

\section{Conclusion}

Based on this experience we believe that colostomy closure can be performed with minimal morbidity, provided a meticulous technique is observed.

Open Access This article is distributed under the terms of the Creative Commons Attribution Noncommercial License which permits any noncommercial use, distribution, and reproduction in any medium, provided the original author(s) and source are credited.

\section{References}

1. Kiely EM, Sparnon AL (1987) Stoma closure in infants and children. Pediatr Surg Int 2:95-97

2. Millar AJK, Lakhoo K, Rode H et al (1993) Bowel stomas in infants and children. A 5-year audit of 203 patients. S Afr J Surg 3:110-113
3. Rees BI, Thomas DFM, Negam M (1982) Colostomies in infancy and childhood. Z Kinderchir 36:100-102

4. Ekenze SO, Agugua-Obianyo NEN, Amah CC (2007) Colostomy for large bowel anomalies in children: a case controlled study. Int J Surg 5:273-277

5. Mollitt DL, Malangoni MA, Ballantine TVN et al (1980) Colostomy complications in children. An analysis of 146 cases. Arch Surg 115:445-458

6. Uba AF, Chirdan LB (2003) Colostomy complications in children. Ann Afr Med 2:9-12

7. Steinau G, Ruhl KM, Hornchen H et al (2001) Enterostomy complications in infancy and childhood. Langenbeck's Arch Surg 386:346-349

8. Das S (1991) Extraperitoneal closure of colostomy in children. J Indian Med Assoc 89:253-255

9. Chandramouli B, Srinivasan K, Jagdish S et al (2004) Morbidity and mortality of colostomy and its closure in children. J Pediatr Surg 39:596-599

10. Miyano G, Yanai T, Okazaki T et al (2007) Laparoscopy-assisted stoma closure. J Laparoendosc Adv Surg Tech A 17:395-398

11. Figueroa M, Bailez M, Solana J (2007) Morbilidad de la colostomia en ninos con malformaciones anorrectales (MAR). Cir Pediatr 20:79-82

12. Nour S, Beck J, Stringer MD (1996) Colostomy complications in infants and children. Ann R Coll Surg Engl 78:526-530

13. Dobe CO, Gbobo LI (2001) Childhood colostomy and its complications in Lagos. East Central Afr J Surg 6:25-29

14. Macmahon RA, Cohen SJ, Eckstein HB (1963) Colostomies in infancy and childhood. Arch Dis Child 38:114-117

15. Rickwood AMK, Hemlatha V, Brooman P (1979) Closure of colostomy in infants and children. Br J Surg 66:273-274

16. Brenner RW, Swenson O (1967) Colostomy in infants and children. Surg Gynecol Obstet 124:1239-1244

17. Pena A, Migotto-Krieger M, Levitt MA (2006) Colostomy in anorectal malformations: a procedure with serious but preventable complications. J Pediatr Surg 41(4):748-756

18. Nichols RL, Broido P, Condon RE et al (1973) Effect of preoperative neomycin-erythromycin in intestinal preparation on the incidence of infectious complications following colon surgery. Ann Surg 178:453-459

19. Ordorica-Flores RM, Bracho-Blanchet E, Nieto-Zermeno J et al (1998) Intestinal anastomosis in children: a comparative study between two different techniques. J Pediatr Surg 33:1757-1759

20. Garcia-Osogobio SM, Takahashi-Monroy T, Velasco L et al (2006) Single-layer colonic anastomoses using polyglyconate 
(Maxon) vs. two-layer anastomoses using chromic catgut and silk. Rev Invest Clin 58:198-203

21. McAdams AJ, Meikle AG, Taylor JO (1970) One layer or two layer colonic anastomoses? Am J Surg 120:546-550

22. Dinsmore JE, Maxson RT, Johnson DD et al (1997) Is nasogastric tube decompression necessary after major abdominal surgery in children? J Pediatr Surg 32:982-985
23. Nelson R, Edwards S, Tse B (2005) Prophylactic nasogastric decompression after abdominal surgery. Cochrane database of systematic reviews (1):CD004929 (online) 